\title{
Handling large variations in mechanics: Some applications
}

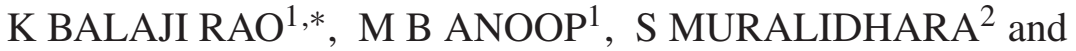 \\ B K RAGHU PRASAD ${ }^{3}$
}

${ }^{1}$ CSIR-SERC, CSIR Campus, Taramani, Chennai 600 113, India

${ }^{2}$ Department of Civil Engineering, BMS College of Engineering, Bangalore 560 019, India

${ }^{3}$ Department of Civil Engineering, Indian Institute Science, Bangalore 560 012, India e-mail: balajiserc1@yahoo.com; smuralidharabms@gmail.com; bkr@civil.iisc.ernet.in

MS received 11 October 2014; revised 17 February 2015; accepted 1 March 2015

\begin{abstract}
There is a need to use probability distributions with power-law decaying tails to describe the large variations exhibited by some of the physical phenomena. The Weierstrass Random Walk (WRW) shows promise for modeling such phenomena. The theory of anomalous diffusion is now well established. It has found number of applications in Physics, Chemistry and Biology. However, its applications are limited in structural mechanics in general, and structural engineering in particular. The aim of this paper is to present some mathematical preliminaries related to WRW that would help in possible applications. In the limiting case, it represents a diffusion process whose evolution is governed by a fractional partial differential equation. Three applications of superdiffusion processes in mechanics, illustrating their effectiveness in handling large variations, are presented.
\end{abstract}

Keywords. Large variations; Weierstrass Random Walk; super diffusion process; alpha-stable distribution; power-law distributions.

\section{Introduction}

As pointed out by Lévy (1937), some of the physical phenomena may evolve in such a way that they tend to be governed by distributions which are having power-law decaying tails and satisfying generalized central limit theorem. This means, there are various basins of attractions for describing the large variations in the observed phenomenon which are still stable (locally perhaps). Rubi (2008) indicated that such situations arise when dealing with molecular dynamics, or in case of mechanics (solid/fluid), when modeling the response at mesolevel in the presence of discontinuities.

In structural mechanics, cases would arise, when there is a sudden change in the trend of evolution of response quantities with the change in the external action/force. Typical examples are

${ }^{*}$ For correspondence 
evolution of strain in reinforced concrete member subjected to axial tension, strain evolution in the tension zone of reinforced concrete flexural member, stiffness degradation of reinforced concrete beam under load control and of a beam-column joint subjected to reverse cyclic loading. In all these cases, the response evolution, at the points of change in trend, which are points of bifurcation, would exhibit large variations compared to other portions of evolution. As was argued in Balaji Rao \& Anoop (2013), at these points, distributions with power-law decaying tails are more appropriate to handle large variations exhibited by the response variables. Large variations are characterized by either infinite variance, and in some cases lack of existence of mean value of the response. In such cases, depending on the value of power law exponent, $\alpha(0<\alpha<2)$, fractional order moments of the response exist. There is a relationship between Madelbrot-Weierstrass function and fractional differential/integral calculus (West et al 2003). The fluctuation-dissipation considerations for such systems would lead to dealing with Weierstrass Random Walk (WRW). A classical one-dimensional stochastic process which exhibits large variations is a Weierstrass Random Walk (WRW). In the limiting case, it represents a diffusion process whose evolution is governed by a fractional partial differential equation (Klafter et al 1996). A good introduction to fractional dynamics and its application to modeling of anomalous diffusion processes are given in Metzler \& Klafter (2004). While superdiffusion processes are finding applications in disciplines such as physical- and biological-sciences, econophysics, their application for engineering systems, to handle large variations, is scantily explored (see Di Paola et al 2013 for some of the applications of deterministic counterpart of anomalous diffusion in structural mechanics).

The aim of this paper is to present the mathematical preliminaries related to WRW that would help in possible applications. While the derivation presented follows closely Metzler \& Klafter (2004), the interpretations presented in this section are those of the authors. The alpha-stable distribution derived from the WRW is applied to describe the variations in surface strains in RC flexural members and in describing the large variations in negative wind pressures on the roofs of low-rise buildings. These two examples are presented only briefly to demonstrate the application possibilities. However, more details of the same are available in Balaji Rao \& Anoop (2013) and Balaji Rao et al (2014). In this paper, for the first time, the application possibility of alpha stable distributions for the determination of critical defect size using AE signals is explored; presented as the third example application.

\section{The Weierstrass Random Walk (WRW) (Metzler \& Klafter 2004)}

In this section, the mathematical preliminaries related to the WRW, required for characterizing the tail behavior of the displacement distribution, are presented.

Consider a random walk on a 1-D lattice with lattice points equi-spaced at ' $a$ '. In a normal random walk problem, the walker/particle will take one step from current position (to the left with probability $q$ and to the right with probability $p$; it is assumed that the system will always change state). The step length is same, and, $p$ and $q$ remain invariant. Application of such random walk problems in engineering has been innumerable (Bogdanoff \& Kozin 1985; Ang \& Tang 1984). The continuous analogue of the normal random walk is Brownian motion. A good review of R\&D studies carried out in the area of Brownian motion is presented by Hanggi \& Marchesoni (2005). The application possibility of Brownian motion process in structural mechanics is well known. In this paper, we consider a special type of random walk in which jump lengths have Bernoulli scaling, with associated probabilities as follows: 
Let $b>1, M>1$ and jump length distribution given as follows. In this context it may be noted that the fundamental step length $=a$; but multiples of ' $a$ ' will be jumped with the following rule:

$$
\rightarrow\left[\begin{array}{l} 
\pm a \text { with probability } C \\
\pm b a \text { with probability } C / M \\
\vdots \\
\pm b^{J} a \text { with probability } C / M^{J}
\end{array}\right] .
$$

This probability structure has the following properties: (a) At any time jumps of different lengths possible thus enabling thorough mixing; the corresponding probabilities scale, (b) The walk makes even remote states, which are otherwise be not possible to reach or almost impossible to reach, now possible to reach, (c) When it makes a large jump, by chance, it can come to lower states since they have higher probabilities of visiting.

If $L$ is the jump length at any time, then, the probability density of function (pdf) of the same is given by

$$
p_{L}(l)=\sum_{J=0}^{\infty} \frac{C}{M^{J}}\left[\delta\left(l-b^{J} a\right)+\delta\left(l+b^{J} a\right)\right] ; \quad-\infty<l<\infty,
$$

where $\delta()$ is the Dirac delta function. The normalization constant $C$ can be computed as follows:

$$
\begin{gathered}
\int_{-\infty}^{\infty} p_{L}(l) d l=1 \\
\int_{-\infty}^{\infty} \sum_{j=0}^{\infty} \frac{C}{M^{J}}\left[\delta\left(l-b^{J} a\right)+\delta\left(l+b^{J} a\right)\right] d l= \\
\sum_{j=0}^{\infty} \frac{C}{M^{J}}\left[\int_{-\infty}^{\infty} \delta\left(l-b^{J} a\right) d l+\int_{-\infty}^{\infty} \delta\left(l+b^{J} a\right) d l\right]=1 \\
C=\frac{1}{2}\left(1-\frac{1}{M}\right)=\frac{M-1}{2 M}
\end{gathered}
$$

Therefore, the pdf of $L$ is given by

$$
p_{L}(l)=\frac{M-1}{2 M} \sum_{J=0}^{\infty} \frac{1}{M^{J}}\left[\delta\left(l-b^{J} a\right)+\delta\left(l+b^{J} a\right)\right] ; \quad-\infty<l<\infty .
$$

The intuitive interpretation of the probability structure (Eq. (5)):

(a) The probability structure chosen for jumps are such that, for large $M$, the random walk will become a simple random walk wherein most jumps occur to the left-and right-neighboring sites with probability $C=1 / 2$.

(b) While the above probability structure allows mixing and possibility of visiting sites which were hitherto unreachable (albeit with smaller probabilities), we have the following scenario:

(i) The possibility of larger jump of length ' $b a$ ' is suppressed by a factor $1 / M$, that of next larger length ' $b{ }^{2} a$ ' by a factor $1 / M^{2}$ and so on. 
(ii) Although the distribution allows jumps of all lengths $a, b a, b^{2} a, \ldots$, their probability decrease by an order of magnitude $M$ (in base $M$ ) if the length of jump increases by an order of magnitude (in base ' $b$ ').

(iii) On the average, ' $M$ ' Jumps of length ' $a$ ' have to be completed before a larger jump can be observed. These ' $M$ ' jumps represent the sites visited which are separated by a distance ' $a$ '. About ' $M$ ' such clusters separated by a distance ' $b a$ ' would occur before a jump of length ' $b^{2} a$ ' occurs. This process would continue leading to formation of clusters within clusters.

\subsection{Condition under which the random walk can develop into a self-similar walk with infinite} hierarchy of clusters

The second moment of jump length, $L$, also can be considered as mean square displacement per step, should become infinite, that is, $\left\langle L^{2}\right\rangle=\infty$, where $<\cdot>$ is the expectation operation.

$$
\left.\begin{array}{l}
<L^{2}>=\int_{-\infty}^{\infty} l^{2} p_{L}(l) d l \\
=\int_{-\infty}^{\infty}\left(\frac{M-1}{2 M}\right) \sum_{J=0}^{\infty}\left(b^{J} a\right)^{2} \frac{1}{M^{J}}\left[\delta\left(l-b^{J} a\right)+\delta\left(l+b^{J} a\right)\right] d l \\
=\frac{(M-1) a^{2}}{2 M} 2 \sum_{J=0}^{\infty} \frac{\left(b^{J}\right)^{2}}{M^{J}} \\
=\frac{(M-1) a^{2}}{M} \sum_{j=0}^{\infty}\left(\frac{b^{2}}{M}\right)^{J}
\end{array}\right\} .
$$

In order that $<L^{2}>$ goes to infinity, $b^{2}$ should be much greater than $M$ and also considerable number of jumps would have taken place. Thus, for $b^{2}>>M, p_{L}(l)$ tends to a stable distribution for large displacements ' $l$ '.

As is known we may not be able to write explicit form of pdf for a stable distribution arising out of a random walk with Bernoulli scaling. However, the characteristic function always exists. Therefore,

$$
\text { Characteristic function of } \left.\begin{array}{rl}
L & =\Phi_{L}(k)=\int e^{i k l} p_{L}(l) d l \\
& =\int_{-\infty}^{\infty} e^{i k l} \frac{M-1}{2 M} \sum_{J=0}^{\infty} \frac{1}{M^{J}}\left[\delta\left(l-b^{J} a\right)+\delta\left(l+b^{J} a\right)\right] d l \\
& =\frac{M-1}{M} \sum_{J=0}^{\infty} \frac{1}{M^{J}} e^{\left(i . k \cdot b^{J} a\right)}
\end{array}\right\} .
$$

Therefore, the characteristic function of displacement, $L$, is given by

$$
\Phi_{L}(k)=\frac{M-1}{M} \sum_{J=0}^{\infty} \frac{1}{M^{J}} \cos \left(k b^{J} a\right) .
$$

This function was invented by Weierstrass (1885) in the second half of $19^{\text {th }}$ century, to provide an example of a function which is continuous everywhere and differentiable nowhere, when 
$b>M$. Equation (8), the characteristic function the stable distribution, satisfies the scaling law as shown here.

$$
\left.\begin{array}{r}
\Phi_{L}(b k)=\frac{(M-1)}{M} \sum_{J=0}^{\infty} \frac{1}{M^{J}} \cos \left(k b^{J+1} a\right) \\
=\frac{(M-1)}{M} \sum_{J=0}^{\infty} \frac{1}{M^{J}} \cos \left(k b^{J} a \cdot b\right)
\end{array}\right\}
$$

Equation (10) is an inhomogeneous "difference" equation, whose solution has two parts: (a) homogeneous part and, (b) inhomogeneous part. That is,

$$
\Phi_{L}(k)=\Phi_{L}^{h}(k)+\Phi_{L}^{i}(k) .
$$

It has been shown in Balaji Rao \& Anoop (2013) that homogeneous part has to exhibit singular behavior to satisfy the condition $\left\langle L^{2}\right\rangle=\infty$, since the inhomogeneous portion contributes to regular variations with finite variance. The latter claim can be shown by seeking the series solution for inhomogeneous part and imposing the consistency condition on coefficients of expansion (with the coefficients of expansion of cosine series), and, using the property of the characteristic function. The resulting solution for inhomogeneous part is given by

$$
\Phi_{L}^{i}(k)=1+\frac{M-1}{M} \sum_{J=1}^{\infty} \frac{(-1)^{J}}{(2 J) !} \frac{(k a)^{2 J}}{\left(1-\frac{b^{2 J}}{M}\right)}
$$

\subsection{Determination of the solution of the homogeneous part}

The difference equation corresponding to the homogeneous part is

$$
\Phi_{L}^{h}(k)=\frac{1}{M} \Phi_{L}^{h}(b k) ; b>1, M>1 .
$$

This equation suggests that the solution sought should be such that functional form of left- and right-hand side functions should be the same and also satisfy the scaling properties. Knowing that the characteristic function is a complex function of a random variable involving $a$ (the basic step length) and $b$ (the modulating factor of step length), the following symmetrical functional form, $\psi($.$) , is assumed.$

$$
\Phi_{L}^{h}(k)=\psi(\exp ( \pm 2 \pi i(\ln |k a| / \ln b))
$$

Hence,

$$
\Phi_{L}^{h}(b k) \propto \psi^{\prime}\left(\exp (2 \pi i) .(\exp ( \pm 2 \pi i(\ln |k a| / \ln b))) \propto \Phi_{L}^{h}(k) .\right.
$$

The functional form $\psi^{\prime}\left(\right.$.) satisfies the relation $\Phi_{L}^{h}(k)=\Phi_{L}^{h}(b k)$. This is periodic function in $\ln k$ with a period of $\ln b$. Letting the function $\psi^{\prime}($.) as $Q(k)$, this function satisfies the property $Q(k)=Q(b k)$. The final homogeneous solution can be written in a power law form as follows:

$$
\Phi_{L}^{h}(k)=|k a|^{\alpha} Q(k) .
$$

The absolute value is considered in the above equation to take into account the fact that the pdf of the displacement is real. It can be shown that the exponent can be obtained from $\alpha=$ $(\ln M / \ln b)$ so that $0<\alpha<2$ and $1<b<\mathrm{M}^{2}$. 
The complete solutions of Eq. (11) can be shown to be

$$
\Phi_{L}(k)=|k a|^{\alpha} Q(k)+1+\frac{M-1}{M} \sum_{J=1}^{\infty} \frac{(-1)^{J}}{(2 J) !} \frac{(k a)^{2 J}}{\left(1-\frac{b^{2 J}}{M}\right)} .
$$

It may be noted that, in Eq. (17), the value of $J$ starts from one as against zero (as in other corresponding equations presented earlier).

- Since $\alpha<2$, the dominant small- $k$ (i.e., large distance) behavior of $\Phi_{L}(k)$ comes from solution of homogeneous part. It scales as $|k|^{\alpha}$, with superimposed log-periodic oscillations.

- For $0<\alpha<2$, the process has infinite variance and thus the process diffuses faster than a normal diffusion process and hence WRW process is a "super diffusion process".

- The small $k$ behavior can be represented by the following characteristic function:

$$
\Phi_{L}(k) \approx 1-c(\alpha)|k a|^{\alpha} \approx e^{-c(\alpha)|k a|^{\alpha}} \text { as } k \rightarrow 0 .
$$

Since WRW is a superdiffusion process governed by the characteristic function Eq. (18), the basic governing differential equation governing the evolution of the pdf of $L$ in space and time is of importance. The resulting diffusion equation (corresponding to the Brownian motion equation in a classical diffusion problem) is a fractional diffusion equation Balaji Rao \& Anoop (2013).

The above characteristic equation and its Fourier transform are used to fit the alpha-stable distribution for large variations arising in three structural mechanics problems in the next section.

\section{Application of superdiffusion process in mechanics}

Efforts are being made at CSIR-SERC, to apply the alpha-stable distributions to describe large variations in the measured surface strains in reinforced concrete flexural members (Balaji Rao \& Anoop 2013) and to characterize large variations in negative wind pressure coefficient on the roofs of low-rise buildings subjected to wind loading (Balaji Rao et al 2014). In both the cases, the models are developed considering the phenomenological aspects. In this paper, only salient results are presented. More details are available in the respective papers. A third application dealing with use of power-law tailed distribution in evolution of the concrete fracture process, leading to estimation of critical defect size using AE data, is also presented.

\subsection{Statistical modeling of surface strains in reinforced concrete flexural members}

To predict/assess the condition of a reinforced concrete (RC) member, prediction of extreme (largest) value of strain is important. Hence, it is important to model the strain as a random quantity taking into account the actual mechanism of cracking and by giving due consideration to the heterogeneity of concrete. One of the outstanding features of the behavior of RC flexural members under the external loading is the emergent structure at different stages of loading. From the results of probabilistic analysis (Prakash Desayi \& Balaji Rao 1987) of average strain, at various stages of loading, it has been found that the average strain, at a given depth, exhibits large scatter, and, the limits (mean $\pm 1.64 \times$ standard deviation) do not enclose the observed range of strain at a given loading stage. These observations suggest that prediction of average strain (compression or tension) itself is beset with large uncertainty.

It may be noted that the physical process of cracking in $\mathrm{RC}$ members is an irreversible process. Hence, the non-equilibrium thermodynamics of irreversible process should be used. At the 
critical points, new dissipative structures form and the type of equilibrium to be considered are far from equilibrium position. Except for the points at which loading drops, which are points of non-equilibrium and far from equilibrium, local equilibrium can be obtained and local stationary states can be defined. However, the points where new emergent dissipative structures form (i.e., new macro-cracks form on the surface of the beam) are far-from equilibrium condition, and the applicability of probability distribution with exponential tails is questionable. Also, the applicability of mean field theory is questionable at the points of unloading (bifurcation), and distributions with heavy tails may be required to describe the large variation phenomenon. It is in this context, alpha-stable distributions are proposed to describe the variations in surface strains near the critical points in the flexural members.

Three reinforced concrete beams (namely KB1, KB2 and KB3) of similar cross-sectional dimensions of $250 \mathrm{~mm} \times 350 \mathrm{~mm}$ and $4.8 \mathrm{~m}$ long were cast and tested in two-point bending over an effective span of $4.2 \mathrm{~m}$. At any given loading stage, 16 strain readings (eight on the west face and eight on the east face) in the constant bending moment region are available for the beams KB1, KB2 and KB3. Using the measured strains, the statistical properties are computed at each stage of loading (using 16 values of measured strain at a given level and at a given stage of loading). The cumulative distribution functions (CDFs) of compression and tension strains are obtained. An alpha-stable distribution is fitted to the observed strains. Other hypothetical distributions considered were normal and Weibull distributions. The results are presented below.

Results: An alpha-stable distribution is fitted to the strains in concrete at the level of reinforcement and at the extreme compression fiber at each loading stage for the beams considered. The parameters of the alpha-stable distributions are estimated using an optimization procedure. Figure 1 corresponds to tensile strains at the level of reinforcement while figure 2 corresponds to strains in extreme compression fibers. From figures 1-2 and other such plots (not presented here) for different loading stages, it is noted that CDF corresponding to the alpha-stable distribution compares with the experimental CDF better than the normal CDF for both compression and tension strains. To examine whether a three parameter Weibull distribution could be a better fit to experimentally observed strains, comparisons of the CDFs with CDFs computed based on measured strains were done. Typical results are shown in figure 3 for beam KB1 (with respect to compression strain). From this figure it is clear that the alpha-stable distribution CDF agrees better with the CDF computed based on measured values, especially in the tails regions - which is of engineering interest, than the CDF of three parameter Weibull distribution.

From the above comparative studies, it is noted that an alpha-stable distribution is better suited (compared to normal distribution) to describe the variations in measured strains in the tail
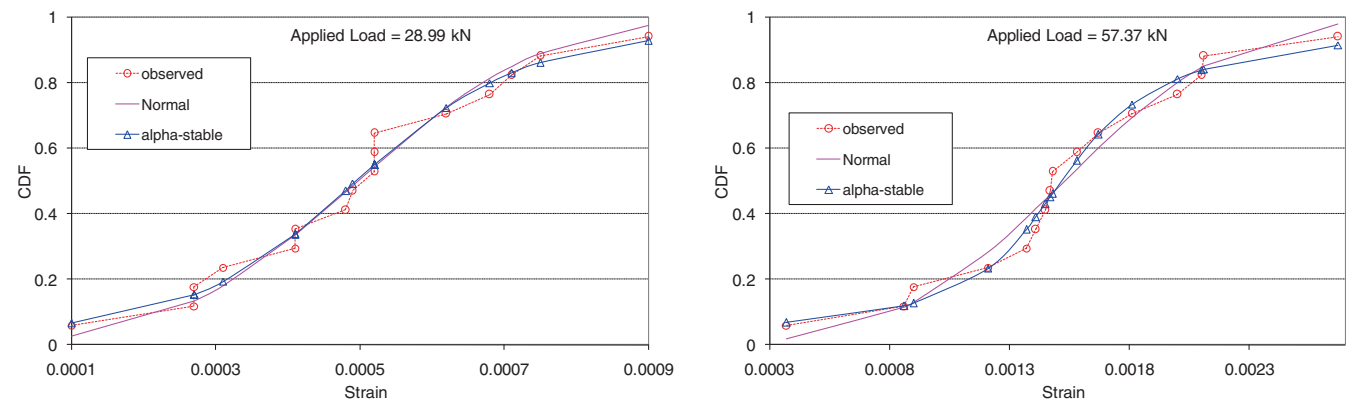

Figure 1. Comparison of CDFs of the surface strains at the level of tensile reinforcement for beam KB1 (Note: observed is same as measured). 

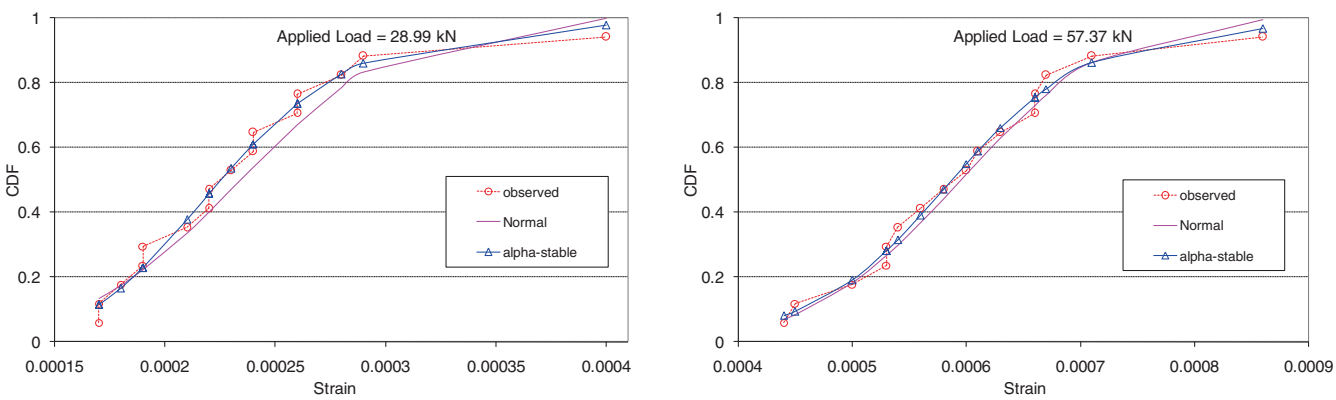

Figure 2. Comparison of CDFs of the surface strains in the compression zone (level 1) for beam KB1 (Note: observed is same as measured).

regions. It may be noted that only the loading stages with emergent structures are considered in the strain analysis. In order to examine this further and to see whether there is any trend in parameters $\alpha$ and $\beta$ of alpha-stable distribution, values of these parameters at different stages of loading for the three beams are plotted in figure 4. From these figures, it is noted that the values of $\alpha$, in almost all cases, are different from 2.0 (representing that the distributions are better represented by alpha-stable distribution).

\subsection{Statistical modeling of negative peak wind pressure coefficients on the roofs of low-rise buildings}

The local environment near the corner of the roof can be modeled as a non-stationary and as a non-equilibrium thermodynamically open system. In such systems, it is known that the physical mechanisms generating the fluctuating forces are distinct from, and independent of, those generating dissipative forces. Hence, there may be no relation between fluctuations and dissipation, and such systems do not approach a thermodynamic equilibrium state asymptotically. More often, in such systems, the energy flux provided by the fluctuations may not be quenched by dissipation mechanism leading to instability (Lindenberg \& West 1990). This will manifest in the form of jumps in the time histories of wind pressure coefficients. To account for these fluctuations in large negative peak pressures, there is a need to use probability distributions with heavy tails to model these pressure fluctuations.
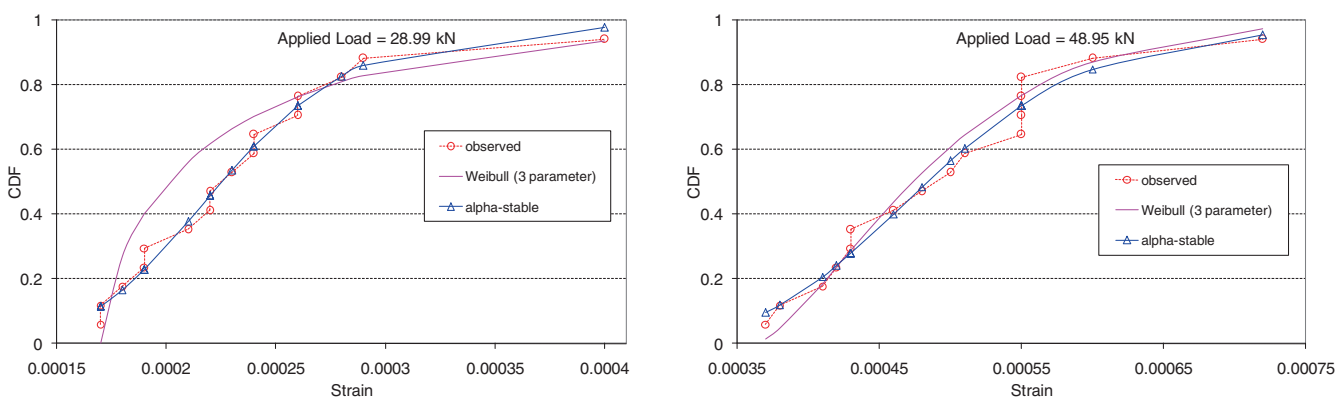

Figure 3. Comparison of CDFs of fitted alpha-stable distribution with Weibull (3 parameter) distribution for measured strains at extreme compression fiber (Level 1) for beam KB1 (Note: observed is same as measured). 

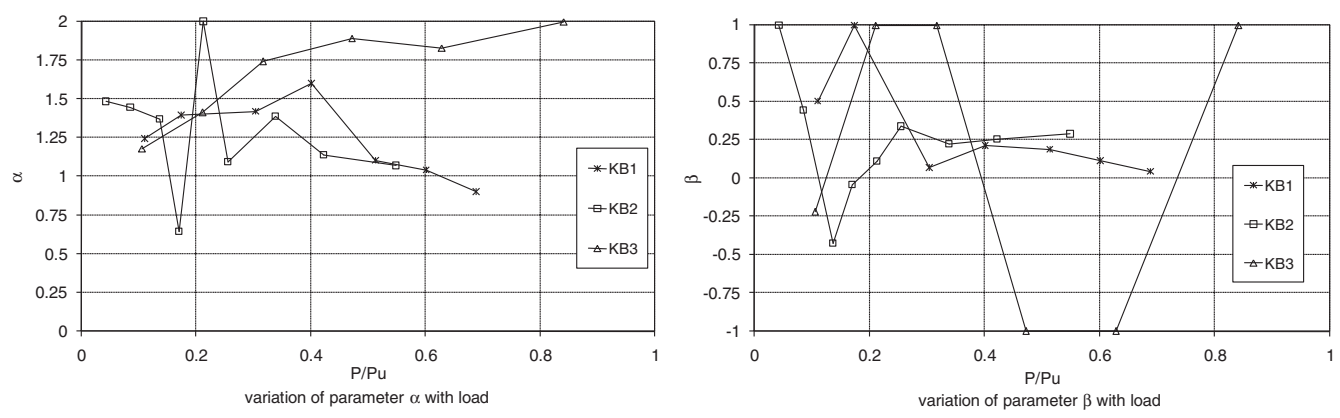

Figure 4. Variation in parameters $\alpha$ and $\beta$ with applied load (for strains at the level of tensile reinforcement).

In this study, an attempt is made to examine the applicability of alpha-stable distribution to model the extreme wind pressure coefficients on the roof corner regions of a low-rise gabled roof building (see figure 5), using wind tunnel experimental data from the aerodynamic database of Tokyo Polytechnic University (TPU 2013). The time histories of wind pressure coefficients corresponding to the measurement taps $72,32,82$ and 51 for $\theta=0^{\circ}$ and $90^{\circ}$, and, taps 72 , 52, 32, 73 and 74 for $\theta=45^{\circ}$, are considered in the study. The 1-second peak negative wind pressure coefficients $\left(\check{C}_{\mathrm{p}}\right)$ are obtained, and the alpha-stable distribution and Gumbel minimum distribution are fitted to $\check{\mathrm{C}}_{\mathrm{p}}$. Here, 1 -second peak corresponds to the 1 second peak in the fullscale.

Results: The values of parameters of the alpha-stable- and Gumbel minimum- distributions, fitted to 1 -second peak negative wind pressure coefficients $\left(\check{C}_{\mathrm{p}}\right)$, are computed. The cumulative distribution functions (cdf) of 1-second peak wind pressure coefficients, for measurement taps 32 , for $\theta=0^{\circ}$ and $\theta=90^{\circ}$ and for the roof pitch angles considered, are shown in figure 6 . From the cdfs shown in figure 6 and similar figures prepared for other measurement taps considered, it is noted that in most of the cases, alpha-stable distribution provides a better estimate of the lower tail portion of peak wind pressure coefficients. It is known that one of the possibilities for the parent distribution which generates an extreme value distribution is that distribution itself (Ang \& Tang 1984). Hence, it is justifiable to make an assumption that the parent distribution of $\check{C}_{\mathrm{p}}$ should have power law decaying tails.

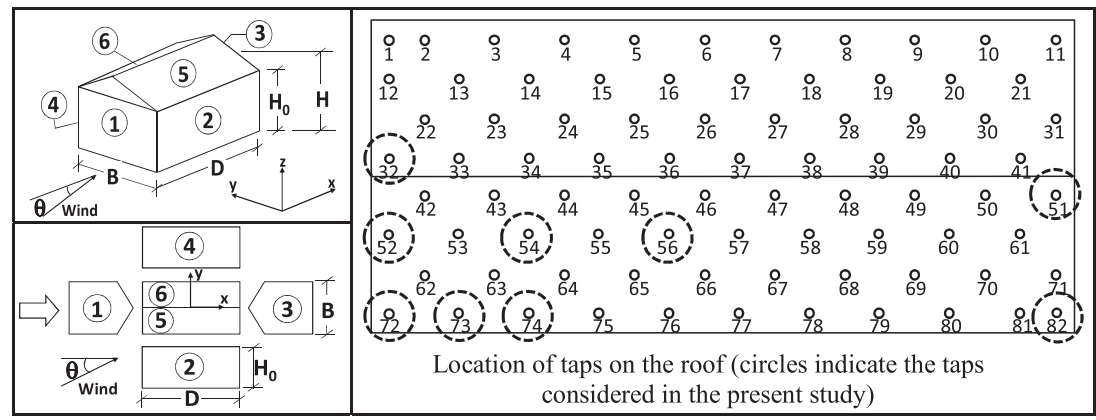

Figure 5. Test model considered and locations of wind pressure taps [TPU (2013)]. 


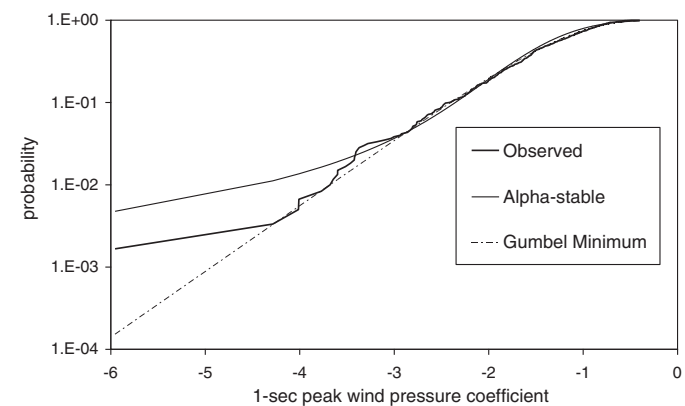

(a) tap 72 , roof pitch angle $=14^{\circ}, \theta=0^{\circ}$

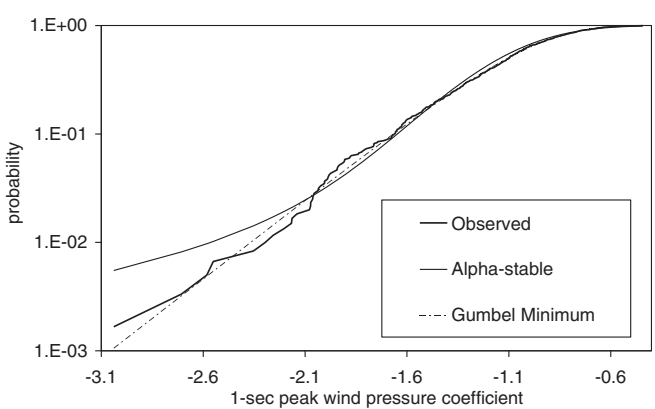

(b) tap 32, roof pitch angle $=14^{\circ}, \theta=90^{\circ}$

Figure 6. Comparison of cdfs of 1-second peak wind pressure coefficients.

To study the efficacy of alpha-stable distribution in modeling the lower tail portion of peak wind pressure coefficients, the values of $\check{C}_{\mathrm{p}}$, typically corresponding to $1 \%$ - and $33 \%$ - probability of non-exceedance are determined. The ratio between observed- and model- of 1-second peak wind pressure coefficients corresponding to $1 \%$ - and $33 \%$ - probability of non-exceedance are shown in figure 7(a) and 7(b). In figure 7(a) and 7(b), 'model' denotes the values of 1-s peak wind pressure coefficients corresponding to the probability of non-exceedance considered assuming $\check{C}_{\mathrm{p}}$ follows alpha-stable-, Gumbel minimum- or Hermite polynomial-based model (where applicable)- distribution. From these figures, it is noted that:

(i) For $1 \%$ probability of non-exceedance (figure 7(a)), the Gumbel minimum distribution gives conservative estimates of $\breve{C}_{p}$ when the values of $\breve{C}_{p}$ are low (less than 1.0). For higher values of $\check{C}_{\mathrm{p}}$, the alpha-stable distribution gives conservative estimates of $\check{C}_{\mathrm{p}}$. The performance of the Hermite polynomial-based model is comparable with that of alpha-stable distribution.

(ii) For $33 \%$ probability of non-exceedance (figure 7(b)), the alpha-stable distribution gives conservative estimates of $\check{C}_{\mathrm{p}}$ regardless of the value of $\check{C}_{\mathrm{p}}$. The Gumbel minimum distribution gives lower estimates of $\check{C}_{p}$ for most of the cases. The performance of the Hermite polynomial-based model is comparatively better than the performances of the alpha-stable distribution and the Gumbel minimum distribution.

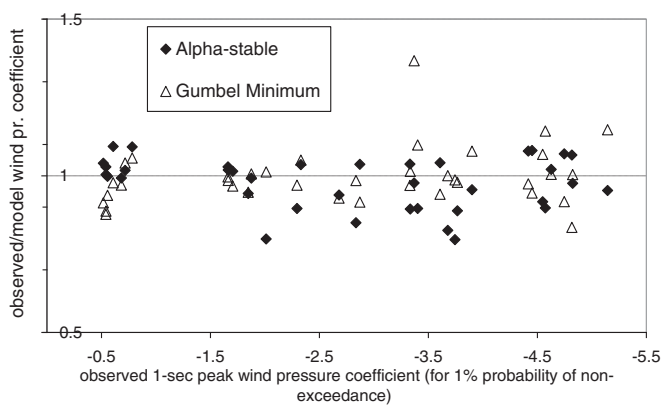

(a) for $1 \%$ probability of non-exceedance

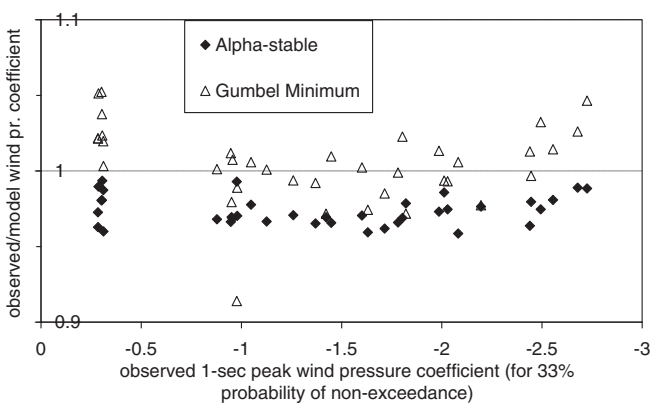

(b) for $33 \%$ probability of non-exceedance

Figure 7. Ratio of observed- to model-1-second peak wind pressure coefficients. 
The above results suggest that to predict maximum suction wind pressure coefficients, alphastable distribution is a suitable candidate distribution. The alpha-stable distribution has an advantage that it is consistent with the physical processes proposed in the literature for explaining large fluctuations at the roof corners. The generality and flexibility offered by alpha-stable distribution makes it a candidate distribution as a single model for predicting the extreme values of negative peak wind pressure coefficients at different regions on the building roof. The alpha-stable distribution can be used to estimate the extreme peak negative wind pressure coefficients.

\subsection{Acoustic emission ( $A E$ ) signals for modelling fracture in concrete}

Recent developments in NDE techniques (such as AE, ultrasonic) have made it possible to study the cracking process in concrete at micro-scale levels (Ohtsu 1996; Rossi et al 1989; RILEM 2010). The characterization of fracture process zone in concrete, a quasi-brittle material, using AE signals is well established (Hadjab 2009). Carpinteri et al (2009a, b), based on laboratory tests on concrete single-edge notched beam specimens, have shown that the $b$-value ${ }^{1}$ of the AE signals can be linked to the value of exponent of the power law form for the tail portion of the probability distribution of crack size, and that the value of exponent of the power law can be interpreted as the fractal dimension of the damage domain/fracture process zone. When AE technique was used to locate damage in RC beams (Carpinteri et al 2009a, b, Colombo et al 2003a, b) and in real life structures (Carpinteri et al 2009a, b), the $b$-value analysis was found to be successful. Hence, AE technique can be used for locating- and assessment (qualitative) ofthe damage. In the present study, the aim is to study the use of power-law-tailed distributions for modeling the defect size in concrete single edge notched beam specimens using AE signals, and to determine the probability of exceeding the characteristic defect size. While the test details, as is required for the present paper, is presented below briefly for the sake of completeness, the determination of critical defect size using power-law tailed distribution is presented for the first time.

AE monitoring (Muralidhara et al 2010): A simply supported plain concrete single edge notched beam specimen subjected to single-point loading is considered (figure 8). The beam has a notch to depth ratio of 0.25 , and notch width of $3 \mathrm{~mm}$. Four sensors were used for the AE acquisition and were arranged on one face of the specimen as shown in figure 8 . The beam was tested under crack mouth opening displacement (CMOD) control.

The load versus CMOD diagram obtained is shown in figure 9. The load-CMOD diagram is divided into 6 regions, which have been indicated in figure 9 using numbers from 1 to 6 . The region 1 spans from the start of the test to the occurrence of the $1^{\text {st }}$ event between the time window $0 \mathrm{~s}-62.58 \mathrm{~s}$. The second region spans from the occurrence of the $1^{\text {st }}$ event to the $100^{\text {th }}$ event between the time window 62.58s-210.62s. The regions 3, 4, 5, and 6 have been divided from the occurrence of the (101-200), (201-400), (401-521), and (522-691) events, respectively between the time windows (210.63s-291.71s), (291.72s-522.99s), (522.99s-870.50s), and (870.51s-2720.28s) respectively. Since there are no AE events in region 1, this region is not considered in the further analysis.

Considering Gutenberg-Ritcher (GR) relations for the AE signals in regions 2-6, the $b$-values have been determined for these five regions, and are given in table 1 . This is used in the present investigation to determine the length of fracture process zone. The characteristic defect size $\left(l_{o}\right)$

\footnotetext{
${ }^{1}$ The $b$-value is the negative gradient of the log-linear AE frequency/amplitude plot.
} 


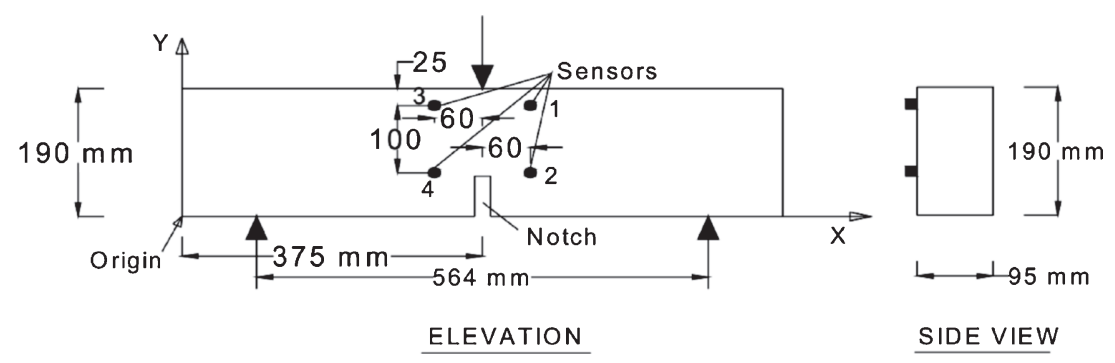

Figure 8. Profile of the beam showing position of the sensors (from Muralidhara et al 2010).

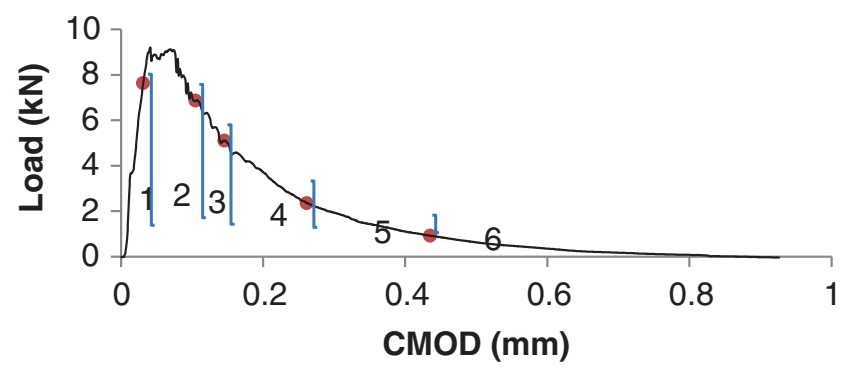

Figure 9. Load vs CMOD.

Table 1. Values of $b$ and $l_{o}$ for the different regions.

\begin{tabular}{lcc}
\hline Region & $b$-value & $l_{o}(\mathrm{~mm})$ \\
\hline 1 & - & - \\
2 & 1.918 & 114.5 \\
3 & 1.657 & 134.3 \\
4 & 1.659 & 141.2 \\
5 & 1.645 & 142.5 \\
6 & 2.425 & 142.5 \\
\hline
\end{tabular}

in the specified region is defined as the defect size which is exceeded only once. For a specific region, $l_{o}$ is determined by first identifying the locations of all the AE events measured during the experimental investigations in that region, and then measuring the distance to the second farthest AE event location from the tip of notch in the depth direction. The values of $l_{o}$ determined for the different regions, based the results of experimental investigations, are given in table 1. From table 1 , it is noted that the characteristic defect size becomes equal to the length of unnotched portion for regions 5 and 6. Hence, in the further analysis, regions 2, 3 and 4 are only considered.

Critical defect size distribution: Let $L$ be the length of defect and $\Delta L$ be the length of unnotched portion, i.e., $\Delta L=d-d_{n}$, where $d$ is the total depth of beam and $d_{n}$ is the depth of the notch. Let $f_{L}(l) s$ be the probability density function of the defect size.

$$
\int_{0}^{\Delta L} f_{L}(L) d l=1 .
$$


One can determine a defect size $l_{o}$ such that on the average only one defect will exceed this value, i.e.,

$$
\mathrm{P}\left(\text { actual defect size out of } \mathrm{N} \text { defects }>l_{o}\right)=1 / N \text {. }
$$

Suppose the defect size in a region is modelled using lognormal distribution. Two different values of mean $(\bar{l})$, namely, $l_{o} / 2$ and $l_{o} / 3$, with a coefficient of variation $(\Omega)$ of 0.20 are considered in the present study. Since the range space of defect size is from 0 to $\Delta L$, a truncated lognormal distribution is considered.

$$
f_{L}(l)=C_{1} \frac{1}{\xi l \sqrt{2 \pi}} e^{-\frac{1}{2}\left(\frac{\ln (l)-\lambda}{\xi}\right)^{2}} ; 0<l \leq \Delta L,
$$
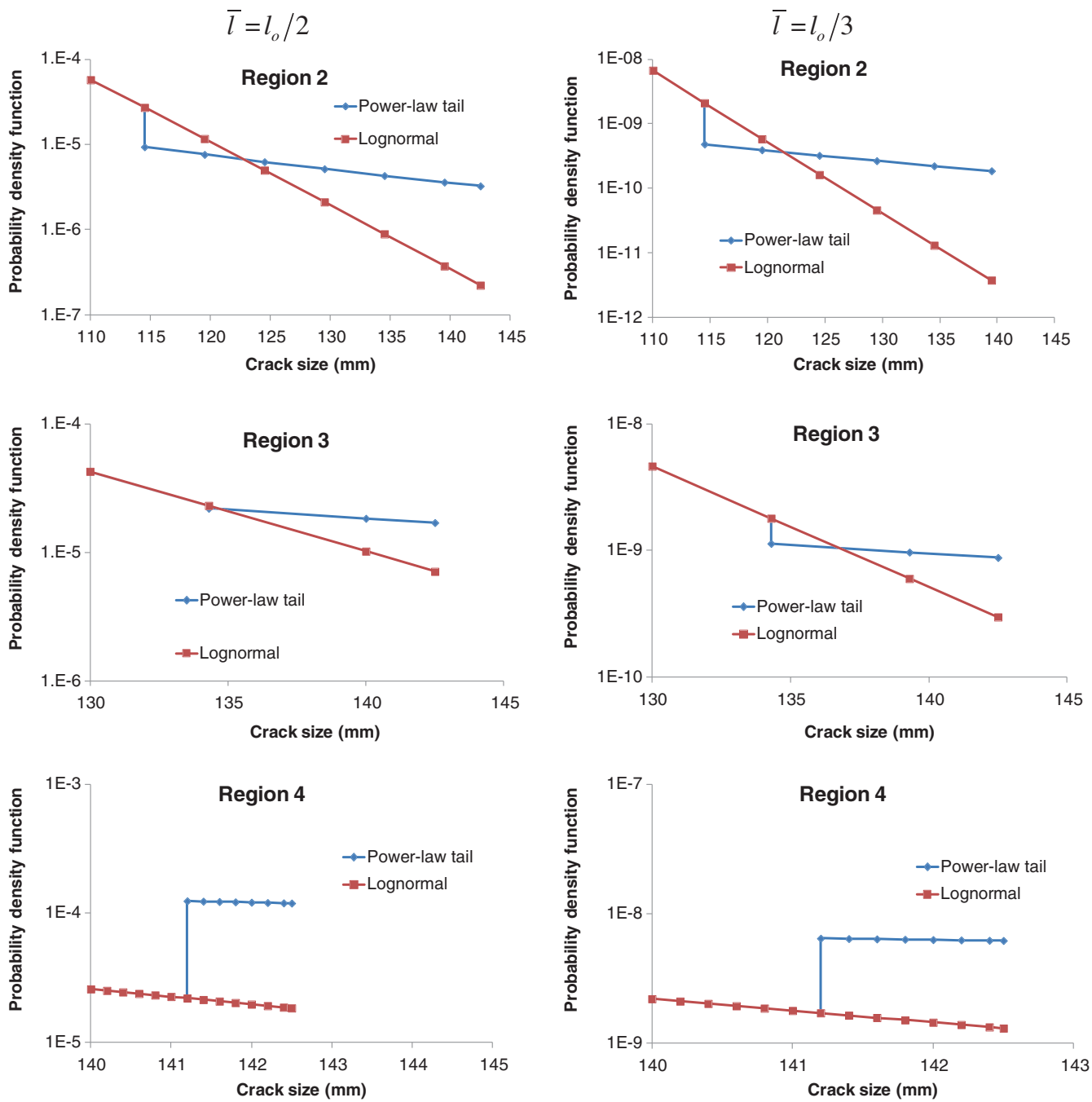

Figure 10. Upper tails of probability distributions of defect size. 
where

$$
\begin{gathered}
C_{1}=\frac{1}{1-\int_{\Delta l}^{\infty} \frac{1}{\xi l \sqrt{2 \pi}} e^{-\frac{1}{2}\left(\frac{\ln (l)-\lambda}{\xi}\right)^{2}}} \\
\xi=\sqrt{\ln \left(1+\Omega^{2}\right)} \\
\lambda=\ln (\bar{l})-\frac{1}{2} \ln \left(1+\Omega^{2}\right) .
\end{gathered}
$$

Considering self-similarity of defects, it can be shown that

$$
f_{L}(l)=\frac{C_{0}}{l^{\gamma+1}} ; l_{o}<l \leq \Delta L,
$$

where $C_{0}$ is constant of proportionality and $\gamma=\frac{\text { fractal dimension of the body }}{\text { degree of self-similarity of defect }}$. Considering the similarity between GR relation and AE activity rate, it can be shown that $\gamma=2 b$.
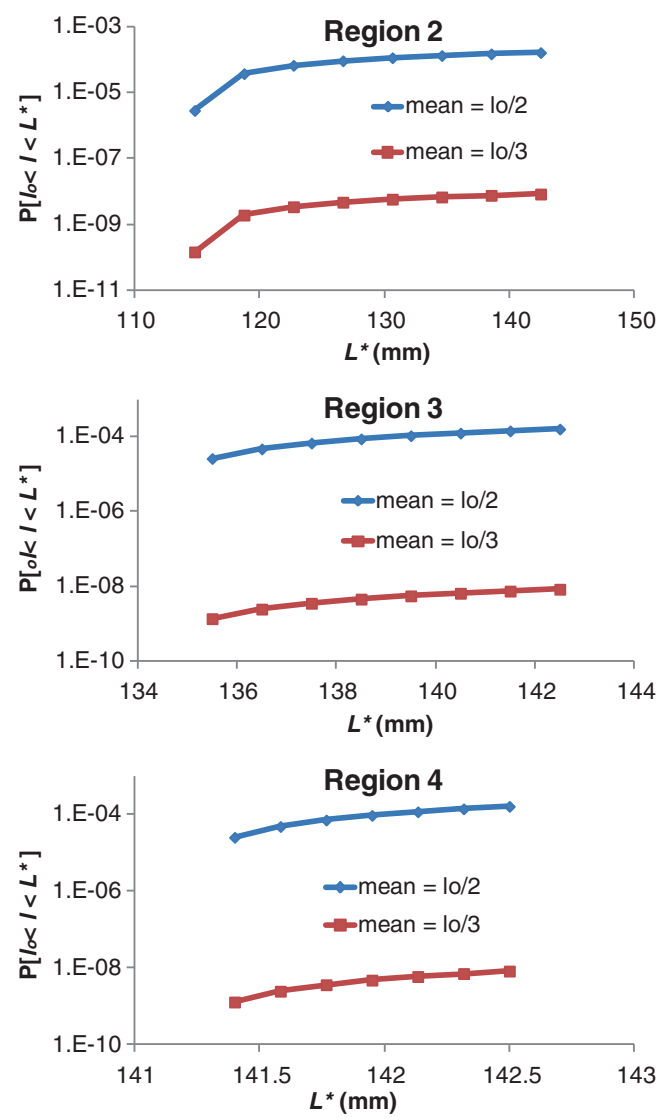

Figure 11. Probability of defect size being greater than $l_{o}$ and less than $L^{*}$. 
Now, considering that the defects follow lognormal distribution over the range 0 to $l_{\mathrm{o}}$ and power-law distribution over the range $l_{\mathrm{o}}$ to $\Delta L$ the value of $C_{0}$ can be determined from

$$
\int_{0}^{l_{o}} C_{1} \frac{1}{\xi l \sqrt{2 \pi}} e^{-\frac{1}{2}\left(\frac{\ln (l)-\lambda}{\xi}\right)^{2}} d l+\int_{l_{o}}^{\Delta l} \frac{C_{0}}{l^{2 b+1}} d l=1 .
$$

The upper tail portions of the probability distributions of defect size, by considering: (i) only lognormal distribution over the entire range, and, (ii) by considering mixture distribution (combination of lognormal- and power-law distributions), are shown in figure 10. From this figure, it is noted that the mixture distribution has a jump at the boundary since the rates at which the tails decay are different for lognormal- and power-law- distributions. From figure 10, it is noted that the use of power-law tail lead to higher probabilities for crack sizes larger than $l_{o}$ when compared to those obtained using exponential tails (as in the case of lognormal distribution). Thus, the use of distributions with power-law tails will help in realistic risk assessment against damage due to fracture. The probabilities that the defect size $(l)$ lie between the characteristic defect size $\left(l_{o}\right)$ and $L^{*}$ (where $l_{o}<L^{*} \leq \Delta L$ ) for the regions 2, 3 and 4, using mixture distribution, are shown in figure 11, for the two mean values considered in this investigation. This type of analysis would help in engineering design decision making.

\section{Summary}

The Weierstrass Random Walk shows promise for modeling the large variations exhibited by some of the physical phenomena in structural mechanics. After presenting the mathematical preliminaries of WRW and superdiffusion/alpha-stable process, the applicability of the same in describing the large variations exhibited in: (a) surface strain development in RC flexural members, (b) negative wind pressures on roofs on low-rise buildings, and (c) critical defect size in concrete, is examined. It may be noted that with the availability of computer programs, the lack of closed form expressions for PDF and CDF of alpha-stable distribution is no longer a bottle neck for its use in engineering applications. Further investigations are being carried out at CSIR-SERC to study the applicability of the alpha-stable distributions for other structural mechanics problems exhibiting large variations.

\section{Acknowledgements}

This paper is being published with the kind permission of the Director, CSIR-Structural Engineering Research Centre, Chennai.

\section{References}

Ang A H S and Tang W H 1984 Probability concepts in engineering planning and design. Decision, risk and reliability. Vol. II, Wiley, New York, NY, USA

Balaji Rao K and Anoop M B 2013 Why do we need probability distributions with fat tails to describe the surface strain evolution in reinforced concrete flexural members? Meccanica 48(6): 1517-1542

Balaji Rao K, Anoop M B, Harikrishna P, Selvi Rajan S and Iyer Nagesh R 2014 Alpha-stable distribution for prediction of negative peak wind pressures on roofs of low-rise building. In: Proceedings of $8^{\text {th }}$ Asia-Pacific conference on wind engineering, December 10-14, 2014, 958-967 
Bogdanoff $\mathrm{J} \mathrm{L}$ and Kozin $\mathrm{F} 1985$ Probabilistic models of cumulative damage

Carpinteri A, Lacidogna G and Puzzi S 2009a From criticality to final collapse: Evolution of the "b-value" from 1.5 to 1.0. Chaos, Solitons \& Fractals 41(2): 843-853

Carpinteri A, Lacidogna G and Niccolini G 2009b Fractal analysis of damage detected in concrete structural elements under loading. Chaos, Solitons \& Fractals 42(4): 2047-2056

Colombo I S, Forde M C, Main I G and Halliday J 2003a AE monitoring of concrete bridge beams in situ. Struct. Eng. 81: 41-46

Colombo I S, Main I G and Forde M C 2003b Assessing damage of reinforced concrete beam using "bvalue" analysis of acoustic emission signals. J. Mater. Civil Eng. ASCE 15: 280-286

Di Paola M, Pinnola F P and Zingales M 2013 A discrete mechanical model of fractional hereditary materials. Meccanica 48(7): 1573-1586

Hadjab H 2009 Fracture process zone in concrete beams: experimental investigation and numerical modelling. In: Proceedings of the SEM annual conference, June 1-4, 2009, Albuquerque, New Mexico, USA

Hanggi P and Marchesoni F 2005 Introduction: 100 years of Brownian motion, Chaos, 15: pp. 026101-1026101-5

Klafter J, Shlesinger M F and Zumofen G 1996 Beyond Brownian motion, Physics Today, February 1996, pp. 33-39

Lévy P 1937 Théorie de l'addition des variables alêalories. Paris: Gauthier-Villars

Lindenberg K and West B J 1990 The nonequilibrium statistical mechanics of open and closed systems. New York: VCH Publishing Inc.

Metzler R and Klafter J 2004 The restaurant at the end of the random walk: Recent developments in the description of anomalous transport by fractional dynamics. J. Phys. A: Math. General 37: R161-R208

Muralidhara S, Raghu Prasad B K, Eskandari H and Karihaloo B L 2010 Fracture process zone size and true fracture energy of concrete using acoustic emission. Constr. Build. Mater. 24: 479-486

Ohtsu M 1996 The history and development of acoustic emission in concrete engineering. Mag. Concrete Res. 48(177): 321-330

Prakash Desayi and Balaji Rao K 1987 Probabilistic analysis of cracking of RC beams. Mater. Struct. 20(6): 408-417

RILEM 2010 Recommendation of RILEM TC 212-ACD: acoustic emission and related NDE techniques for crack detection and damage evaluation in concrete - Test method for classification of active cracks in concrete structures by acoustic emission. Mater. Struct. 43: 1187-1189

Rossi P, Robert J L, Gervais J P and Bruhat D 1989 Acoustic emission applied to study crack propagation in concrete. Mater. Struct. 22: 374-384

Rubi J M 2008 The long arm of the second law. Scientific American 299: 62-67

TPU 2013 Aerodynamic database of low-rise buildings. Tokyo Polytechnic University, 2013, http://www. wind.arch.t-kougei.ac.jp/system/eng/ (last accessed: 27 August 2013)

Weierstrass K 1885 Über die analytische Darstellbarkeit sogenannter willkürlicher Functionen einer reellen Veränderlichen. Gesammtsizung 9

West B J, Bologna M and Grigolini P 2003 Physics of fractal operators. Institute for Nonlinear Science: Springer-Verlag 